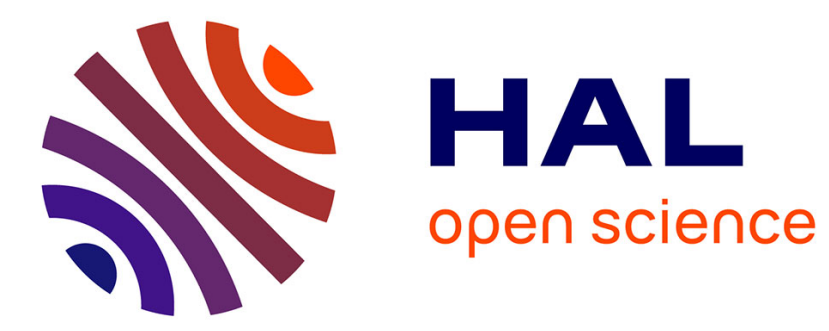

\title{
Partially Observed Objects Localization with PCA and KPCA Models
}

\author{
Vincent Guilloux, Barbara Romaniuk, Michel Desvignes, Marie-Josèphe \\ Deshayes
}

\section{- To cite this version:}

Vincent Guilloux, Barbara Romaniuk, Michel Desvignes, Marie-Josèphe Deshayes. Partially Observed Objects Localization with PCA and KPCA Models. 6th IEEE Southwest Symposium on Image Analysis and Interpretation, 2004, Lake Tahoe, Nevada, United States. pp.80-84. hal-00843457

\section{HAL Id: hal-00843457 \\ https://hal.science/hal-00843457}

Submitted on 12 Jul 2013

HAL is a multi-disciplinary open access archive for the deposit and dissemination of scientific research documents, whether they are published or not. The documents may come from teaching and research institutions in France or abroad, or from public or private research centers.
L'archive ouverte pluridisciplinaire HAL, est destinée au dépôt et à la diffusion de documents scientifiques de niveau recherche, publiés ou non, émanant des établissements d'enseignement et de recherche français ou étrangers, des laboratoires publics ou privés. 


\title{
Partially Observed Objects Localization with PCA and KPCA Models
}

\author{
V. Guilloux, B. Romaniuk*, M. Desvignes**, MJ. Deshayes *** \\ ${ }^{*}$ GREYC Image, 6, Bd Maréchal Juin, 14050 Caen Cedex, France; ${ }^{* *}$ LIS-ENSERG, 961, rue de la Houille \\ Blanche, BP 46, 38402 Saint Martin d'Hères Cedex, France; ${ }^{* * *}$ Société TCI, http://www.cranexplo.com \\ Barbara.Romaniuk@greyc.ismra.fr, michel.desvignes@lis.inpg.fr
}

\begin{abstract}
In this paper, we deal with the problem of partially observed objects. These objects are defined by sets of points and their shape variations are represented by a statistical model. We present two models in the paper: a linear model based on PCA and a non-linear model based on KPCA. The present work attempts to localize non visible parts of an object, from visible parts and from model, explicitly using the variability represented by the model. Both are applied to the cephalometric problem with good results.
\end{abstract}

\section{Introduction}

During the past decade, there has been a lot of work in shape based approaches, for segmentation, registration or identification tasks. In fact, any application where the geometric comparison of objects is required needs a shape analysis. The pioneers of shape analysis are Kendall [1] and Bookstein [2]. In these works, shape is defined as the remaining information after alignment (rotation, translation, scaling) between two objects. In image analysis, Pentland [3] has defined modal analysis and a similar idea has been used by Cootes [4] in the Active Shape Model (ASM) and Active Appearance Model (AAM). They both involve a Principal Component Analysis (PCA) to build a statistical shape model. In this model, the mean object and the variation around this mean position are both represented. AAM was tested by Hutton [6] for the cephalometric problem without sufficient accuracy.

Some works on Kernel PCA [5] are very close to our method. Briefly, Kernel PCA maps non-linearly the input data in a Feature Space ( $F$-Space). PCA is then performed in the $F$-Space. The non linear principal components are given by the eigenvectors corresponding to the largest eigenvalues. In a localization problem, mean shape in the $F$-space must be back-projected in the input space. The choice of the mapping and the back projection are difficult problems and are still open issues.

In this paper, we address the problem of retrieving the position of non visible parts of a partially visible object. The key idea is to use the authorized variation around the mean shape of the model to localize these parts. We present a comparison of one linear method based on PCA representation to another based on KPCA representation. Both are applied to the cephalometric problem.

\section{Methods}

In the cephalometric problem, orthodontists have annotated cephalograms with landmarks (cephalometric points) on a training set of radiographs. We also use an a-priori knowledge, which is an unknown spatial relation between the cranial contour and the cephalometric points. The main problem in the cephalometric analysis is to discover this relation. Fortunately, the cranial contour can be automatically detected and extracted from the image, and then sampled (6 points). Our training set of points is composed of the cephalometric points and the sampled version of the cranial contour. From this dataset, a mean shape model is computed. To retrieve landmarks on a new image, the cranial contour is detected and sampled, cephalograms are registered and the mean shape model is used to estimate the position. 


\section{$2.1 \quad$ Linear PCA model}

The linear PCA method defined here is an elegant way to take into account spatial relations between landmarks and between landmarks and contour, and can also estimate the unknown part (cephalometric points) of the partially visible or occulted model (cranial contour).

Let $X_{i}=\left(x_{1 i}, y_{1 i}, \cdots, x_{n i}, y_{n i}\right) \in \mathbb{R}^{2 n}$ be the locations of the $n$ cephalometric points on the $i^{\text {th }}$ cephalogram, $C_{i}$ be the locations of the $m$ points of the sampled cranial contour on the $i^{\text {th }}$ cephalogram, and $T_{i}=\left(X_{i}, C_{i}\right)$ the concatenation of $X_{i}$ and $C_{i}$.

Using PCA, we can write: $T_{i} \approx \bar{T}+\Phi b$, where $\bar{T}=\frac{1}{p} \sum_{i=1}^{p} T_{i}$ is the mean shape of the pattern, $\Phi=\left(\phi_{1}|\cdots| \phi_{t}\right)$ is a $(n+m) \times t$ matrix composed with the eigenvectors of the $(n+m) \times(n+m)$ covariance matrix $S$ of the centered data $S=\frac{1}{p-1} \sum_{i=1}^{p}\left(T_{i}-\bar{T}\right)\left(T_{i}-\bar{T}\right)^{t}$ and $b$ is a vector of dimension $t: b=\Phi^{t}\left(T_{i}-\bar{T}\right)$.

The dimension $t$ of the vector $b$ is the number of eigenvectors with the largest eigenvalues. In classical use of PCA, $t$ is chosen by $\sum_{i=1}^{t} \lambda_{i} \geq 0.95 \sum_{i=1}^{n+m} \lambda_{i}$, i.e. only eigenvectors that sufficiently explain the standard deviation are kept. The vector $b$ of dimension $t$ is a good approximation for the original dataset and any set of $n+m$ points can be represented or retrieved with the $t_{t<n+m}$ values of the vector $b$. PCA can then be seen as a denoising method.

The original idea of the paper is the following one: if some information is present, if some points (saying $t=n$ points) are known, the remaining unknown $m$ points can be determined using PCA, under the hypothesis that the first $n$ eigenvalues of the covariance matrix explain the training dataset.

Let $C=(x, y)$ be the known and visible points (cranial contour in cephalometry) and $X$ be the unknown and obscured points (cephalometric points). Without any approximations, we can write:

$$
\left[\begin{array}{c}
C_{1} \\
\vdots \\
C_{n} \\
X_{1} \\
\vdots \\
X_{m}
\end{array}\right]=\left[\begin{array}{c}
\bar{C}_{1} \\
\vdots \\
\overline{\bar{C}}_{n} \\
\bar{X}_{1} \\
\vdots \\
\bar{X}_{m}
\end{array}\right]+\left[\begin{array}{ccc}
\Phi_{1,1} & \cdots & \Phi_{1, n+m} \\
\vdots & \ddots & \vdots \\
\Phi_{n+m, 1} & \cdots & \Phi_{n+m, n+m}
\end{array}\right]\left[\begin{array}{c}
b_{1} \\
\vdots \\
b_{n} \\
b_{n+1} \\
\vdots \\
b_{n+m}
\end{array}\right]
$$

This is a linear system with $n+m$ equations and $n+2 m$ unknown $\left(X_{1}, \cdots, X_{m}, b_{1}, \cdots, b_{m+n}\right)$ that cannot be resolved. Since PCA represents the dataset with less values, we can write, using $t=n$, the unknown vector $\left(b_{1}, \cdots, b_{n}, X_{1}, \cdots, X_{m}\right)$ in the following system. Notice that if we choose $t<n$, the system becomes overdetermined $(t<n)$, and a least square method can be used to resolve the system:

$$
\left\|\left[\begin{array}{c}
b_{1} \\
\vdots \\
b_{n} \\
X_{1} \\
\vdots \\
X_{m}
\end{array}\right]-\left[\begin{array}{ccc}
\Phi_{1,1} & \cdots & \Phi_{1, n} \\
\vdots & \ddots & \vdots \\
\Phi_{n+m, 1} & \cdots & \Phi_{n+m, n}
\end{array}\right]\right\|\left(\left[\begin{array}{c}
C_{1} \\
\vdots \\
C_{n} \\
0 \\
\vdots \\
0
\end{array}\right]-\left[\begin{array}{c}
\bar{C}_{1} \\
\vdots \\
\bar{C}_{n} \\
\bar{X}_{1} \\
\vdots \\
\bar{X}_{m}
\end{array}\right] \|^{2}\right.
$$

In this framework, a linear approximation of spatial relations between known and unknown points are explicitly determined from the eigenvectors of the covariance matrix. These spatial relations are used to localize the non visible parts of an object.

\section{$2.2 \quad$ KPCA Non Linear model}

In this model, the initial data are projected in a new space, with a non-linear mapping $\varphi$. PCA is then computed in this feature space $F$, as done with linear model. KPCA has a formulation which uses only dot product and saves computer time. KPCA employs Mercer Kernels, such as gaussian or polynomial kernels, instead of carrying out the mapping explicitly. Thus, when we have n samples $X_{i}$, the principal components $\beta_{i}$ in the KPCA space of an initial vector $X$, are given by: $\beta_{k}=\sum_{i=1}^{n} \alpha_{i}^{k} K\left(X_{i}, X\right)$, where $\alpha^{k}$ are the eigenvectors of the kernel matrix $K\left(X_{i}, X_{j}\right)=\varphi\left(X_{i}\right) \cdot \varphi\left(X_{j}\right)$. 
The reconstruction of the initial value $X$ from the principal components $\beta_{i}$ is a difficult problem. If the mapping is known, the $\varphi$-image $P_{n}(X)$ of $X$ in the feature space is defined by: $P_{n}(X)=\sum_{i=1}^{n^{\prime}} \beta_{k} V^{k}$, where $V=\sum_{i=1}^{n} \alpha_{i} \varphi\left(X_{i}\right)$.

To reconstruct in the initial space, we can use several methods: firstly, we can compute the mapping $\varphi$ explicitly if it is known, compute the PCA, and use the linear method;

secondly, we can use the pre-image $P_{n}(X)$ and find the vector $Z$ in the initial space that minimizes $m_{1}(Z)$ :

$m_{1}(Z)=\left\|\varphi(Z)-\sum_{i=1}^{n^{\prime}} \beta_{i} V^{i}\right\|^{2}$, where $n^{\prime}$ is the number of conserved nodes;

thirdly, we can find $Z$ such that the principal components of $Z$ are very closed to $\beta_{i}$ and minimize $m_{2}(Z)$ :

$m_{2}(Z)=\left\|\left(\sum_{i=1}^{n^{\prime}} \alpha_{i} K\left(X_{i}, Z\right)-\beta\right)\right\|^{2}$, where $\beta$ is a vector of $\beta_{k}$ and $\alpha_{i}$ a vector of $\alpha_{i}^{k}$;

fourthly, we can rewrite this last function and minimize a function $m_{3}(Z)$ :

$m_{3}(Z)=\left\|\left(K\left(X_{j}, Z\right)-\sum_{i=1}^{n^{\prime}}\left(\alpha_{i}[\lambda] \beta\right)\right)\right\|^{2}$, where $[\lambda]$ is a diagonal matrix containing the eigenvalues of the kernel matrix.

For pattern recognition, some parts of the object are unknown. In our framework, the first coordinates of the vector $Z$ are known and are the visible parts of the object. The last coordinates of the vector $Z$ are the non visible parts and are the unknown points. In the expressions to minimize, $\beta_{i}$ are also unknown. The problem is now to determine the values $\beta_{i}$ and the unknown part of $Z$.

\section{Experiments}

To test the feasibility of the algorithm, we have run several toys and real world experiments. We have compared the distance between the calculated points and the real points, using the 5 different methods, with a polynomial kernel for KPCA.

We have simulated an object defined by 9 points. Thereafter, 2 points were suppressed on an image, and we have built these points with the proposed methods by minimization of mi. Linear are less accurate than non-linear methods, but minimization sometimes falls in local minima. However, as the simulated data are really nice, errors are less than $1 \%$ with non-linear methods.

On the cephalometric problem, the cranial contour is approximated by 6 points, and we have tested the methods on the reconstruction of 3 cephalometric points. The models are built with 80 radiographs. We use a leave-one out approach to test the accuracy of the models. The linear model is less accurate than non-linear models with an average error less than $2 \%$.

\section{Conclusion}

In this paper, we have presented and compared several methods to reconstruct non visible parts of an object with a statistical model. The statistical framework offers an elegant way to solve this problem, using the authorized variability by the model. The classical linear model is less accurate than KPCA models, even if some difficulties remain from a numerical point of view.

\section{References}

[1] D.G. Kendall. Shape manifold, Procrustean metrics and complex projective spaces. Bull. London Math. Soc., Vol. 16, pp. 81-121, 1984.

[2] F. L. Bookstein. Principal Warps: Thin-Plate Splines and the Decomposition of Deformations. IEEE PAMI, Vol. 11 (6), pp. 567-585, 1989.

[3] S. Sclaroff, A.P. Pentland. Modal Matching for Correspondence and Recognition. IEEE Transactions on PAMI, Vol. 17(6), pp. 545-561, 1995.

[4] T.F.Cootes, G.J. Edwards, C.J.Taylor. Active Appearance Models IEEE PAMI, Vol. 23 (6), pp. 681-685, 2001.

[5] S. Mika et al. Kernel PCA and de-noising in feature spaces. Advances in Neural Information Processing Systems, Vol. 11, pp. 536-542, 1999.

[6] T.J. Hutton, S.Cunningham, P. Hammond. An Evaluation of Active Shape Models for the Automatic Identification of Cephalometric Landmarks. European Journal of Orthodontics, Vol. 22(5), pp. 499-508, 2000. 